\title{
Automatic MHT Method Based on Target Characteristics Applied in the Small Space Targets
}

\author{
Qian ZHAO ${ }^{1, a}$, Zhenhong NIU ${ }^{1}$,Lian XUE ${ }^{1}$, Xin LIU ${ }^{1}$,Jianhua $\mathrm{LI}^{1}$ and Zhifeng $\mathrm{LI}^{1}$ \\ ${ }^{1}$ National Key Laboratory of Science and Technology on Test Physics \& Numerical Mathematics, China
}

\begin{abstract}
The Multi-Hypothesis Tracking (MHT) method is one of the most promising approaches to realize automatic tracking of small and dim targets in complex space scene. However, the number of hypotheses generated by this algorithm has an exponentially increasing relationship with the number of trajectories, measurement data, and the number of image frames. In the paper, the automatic detection and tracking method of judging the confidence coefficient of the trajectory obtained by the MHT which is based on the characteristics of dim targets, has been realized in the IRST system. The performance of this method is greatly improved.
\end{abstract}

\section{INTRODUCTION}

Automatic tracking technology based on image is one of the important research inspects of Machine Vision Application, and key technology of automatic target detection. It has been widely applied in aerospace systems. Infrared Search and Track (IRST) based on obtaining $2 \mathrm{~d}$ infrared images, has the advantages of large field of view, small size, and working all day to be used Detecting and tracking space targets by receiving infrared radiation from targets and background. However, it is commonly used in space craft to realize automatic detection of target for Engineering Applications. And image noise, spatial illumination, target motion and other factors have always plagued the realization of target automatic tracking. Difficulties in engineering implementation are reflected in three aspects: 1) The system has a large amount of image data, which makes it difficult for its hardware equipment to complete the real-time detection and tracking of the target. 2) The working environment of the system is complex, the image interference area is large and the SNR is low. 3) Infrared small and dim targets for obtaining images through the IRST have no obvious geometric features [1].

The Multi-Hypothesis Tracking (MHT) method which is first proposed by D. B. Reid [2][3], has been commonly used in target tracking studies based on 2D image. Based on this method, it is to create multiple hypothetical trajectories, calculate the prior probability of each hypothesis, and delete the hypothetical trajectories of low probability. Finally, the correct trajectory can be found based on the measurement point. Although MHT is one of the most promising approaches to solve the problem of tracking multiple targets in complex scenarios, the number of hypotheses generated by this algorithm has an exponentially increasing relationship with the number of trajectories, measurement data, and the number of image frames. Therefore, based on this algorithm, multi-objects detecting and tracking is difficult to realize. Based on the feature of dim targets, the automatic detection and

\footnotetext{
${ }^{a}$ Corresponding author : bestcherryzhaoqian@163.com
} 
tracking method of judging the confidence coefficient of the trajectory obtained by the MHT is proposed in paper. This method used in the real-time infrared image processing system, has been realized the automatic tracking of dim space target based on infrared image.

\section{SYSTEM OVERVIEW}

IRST on the spacecraft platform is composed of the IR imaging detector and the real-time infrared image processing system. In complex scenario, the IR imaging detector collects infrared images containing the target, and then transmits it through the connecting cable to the infrared image realtime processing system for processing. The FPGA of infrared image real-time processing system realizes the data stream scheduling and image preprocessing. Meanwhile, DSP accepts the image data from FPGA to complete the complex image processing.

In the space scene, IRST work process is that system scans the certain field according to the prior information, and transmits image data to the infrared image real-time processing system for processing. Firstly, IRST detects and select suspected tracking target. Then the system which is derived from the automatic detection and tracking method, determines the location and size of the target to be detected, and calculates the moving direction and speed information of the target to the spacecraft platform. Finally, the platform controls the detector to track the moving target and keeps target in the field of the detector. During the process, the platform performs target position correction at any time. The working process of IRST is shown in Figure 1.

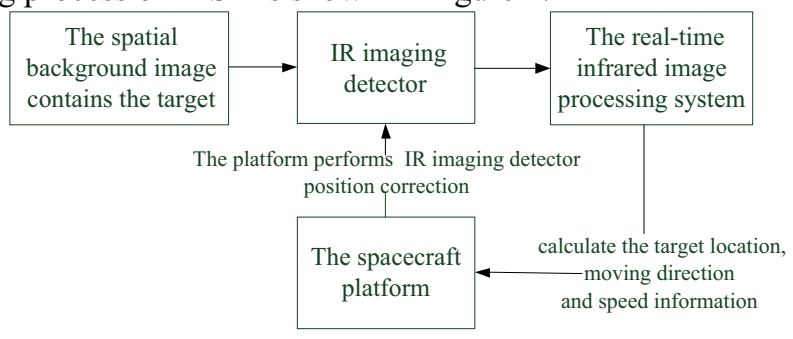

Figure 1. The working process of IRST

\section{MRTHOD IMPLEMENTATION}

The target detection and tracking methods is divided into Detect-before-track (DBT) and Trackbefore-detect (TBD). Currently, the TBD method is mainly used to detect the small and dim targets with low SNR. The principle of TBD is not to judge the target, but to track the possible trajectory of the target in the image, and then to complete the threshold decision through a series of calculations [4]. In all methods conforming to the TBD principle, MHT is the classic algorithm to solve the detection problem of small and dim moving targets in complex scenarios.

\subsection{The principle of MHT}

In the MHT, it is assumed that the maximum range of motion between two adjacent frames is not more than $\mathrm{N}$ pixels. From time $\mathrm{t}$, the target at point $\mathrm{A}$ may appear at any point $\mathrm{B}$ in the square field which is centered at point $\mathrm{A}$ and $2 \mathrm{~N}+1$ for the side. The target has a total of $(2 \mathrm{~N}+1)^{2}-1$ possible trajectories from point A to point $\mathrm{B}$. At $\mathrm{t}+2$, the target moves from any point in $(2 \mathrm{~N}+1)^{2}-1$ at time $\mathrm{t}+1$ to point $\mathrm{C}$. There is a total of $(2(2 \mathrm{~N}+1)-1)^{2}-1$ possible trajectories. From these points, a new trajectory is formed. This is the root cause of the explosion of the target trajectory as the number of image frames increases. According to the reference, the boundary parameters $a, b, \lambda^{\prime}$ and the series estimation parameter $\mathrm{K}^{\prime}$ are used as the threshold parameters in the MHT as the hypothesis trajectory inspection decision [1][2][3]: 


$$
\begin{array}{ll}
H_{0}: x(i, j, k)=w(i, j, k) & \text { There is no target } \\
H_{1}: x(i, j, k)=\mathrm{s}+w(i, j, k) & \text { There is a target }
\end{array}
$$

If $\mathrm{k}<\mathrm{K}^{\prime}$, the decision is as follows:

$$
\left\{\begin{array}{l}
\sum_{i=1}^{k} x_{i} \geq a \quad \text { Judgment for } \mathrm{H}_{1} \\
\sum_{i=1}^{k} x_{i} \leq b \quad \text { Judgment for } \mathrm{H}_{0} \\
b \leq \sum_{i=1}^{k} x_{i} \leq a \quad \text { Wait for the next frame to continue to judge. }
\end{array}\right.
$$

If $\mathrm{k}=\mathrm{K}^{\prime}$, the decision is as follows:

$$
\left\{\begin{array}{l}
\sum_{i=1}^{K^{\prime}} x_{i} \geq \lambda^{\prime} \quad \text { Judgment for } \mathrm{H}_{1} \\
\sum_{i=1}^{K^{\prime}} x_{i} \leq \lambda^{\prime} \quad \text { Judgment for } \mathrm{H}_{0}
\end{array}\right.
$$

The above four parameters are defined as:

$$
\begin{gathered}
a=\ln \left(\frac{1-\left(1-c_{1}\right) \beta^{\prime}}{\left(1-c_{0}\right) \alpha^{\prime}}\right) \\
b=\ln \left(\frac{\left(1-c_{1}\right) \beta^{\prime}}{1-\left(1-c_{0}\right) \alpha^{\prime}}\right) \\
K^{\prime}=\left(\Phi^{-1}\left(c_{0} \alpha^{\prime}\right)+\Phi^{-1}\left(c_{1} \beta^{\prime}\right)\right)^{2}\left(\frac{\sigma}{s-\mu_{0}}\right)^{2} \\
\lambda^{\prime}=\sqrt{K^{\prime}}\left(s \Phi^{-1}\left(c_{0} \alpha^{\prime}\right)+\mu_{0} \Phi^{-1}\left(c_{1} \beta^{\prime}\right)\right)\left(\frac{\sigma}{s-\mu_{0}}\right)
\end{gathered}
$$

\subsection{Adaptive target optimal track extraction method based on target feature constraint optimization}

When tracking targets and multiple interfering targets enter site of the detector at the same time, even if the 2.1 decision condition is used, a large number of candidate trajectories remain, which requires the real-time infrared image processing system to provide large memory and computational overhead. This method relies too much on current data and ignores the use of other image sequence information. At the same time, in the process of track association, there may be several situations such as trajectory bifurcation and trajectory crossing. When multiple measuring points fall into the data association area of a certain trajectory, the trajectory bifurcation occurs;

When a measured data falls within a data association region of multiple trajectories, the trajectory crossing will occur. When a single independent data point appears, a new track may occur. This process is shown in Figure 2. 


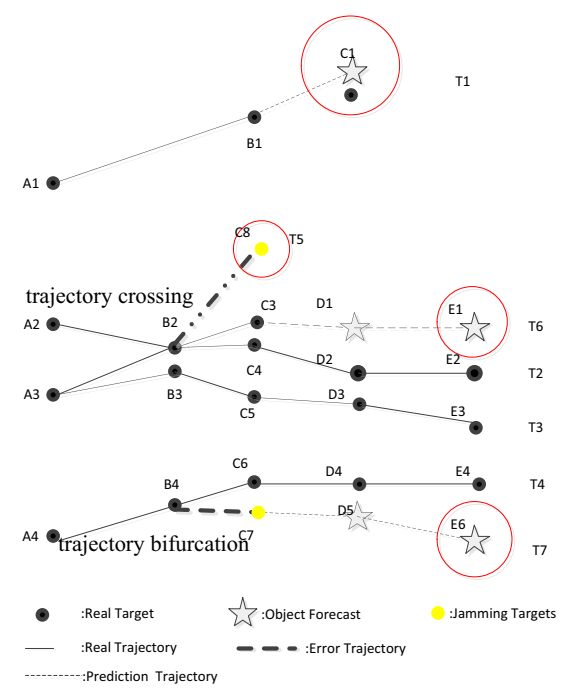

Figure.2. The Process of Track Association

As shown in the figure, multiple error trajectories are obtained through the MHT method, leading to the increase of false alarm rate in the automatic tracking process. Therefore, this paper proposes the adaptive target optimal track extraction method based on target feature constraint optimization. By calculating the confidence of each track, the target motion parameter estimation and trajectory extraction accuracy are improved [5].

For multi-target tracking in IRST, besides the target location information, the target gray information and speed information can be used. At the same time, the speed of movement between the target to be tracked and the target of interference is also different. Therefore, the trajectory judgment criterion of this method is:

(1)If the gray value of the measurement point is close to the gray value of the target, the probability of this measurement point is high.

(2)In the same trajectory, if the measurement point's velocity consistency with the adjacent frame is good, the trajectory is the probability of continuation of the same moving target.

On the contrary, the trace confidence coefficient is low.

After the real-time infrared image processing system accumulates multiple frame information for each currently recorded trajectory, the trace confidence coefficient is automatically evaluated based on the target feature. During the system working, the trajectory of confidence coefficient below the threshold is deleted at any time. According to the reference, the logarithmic form of the trajectory evaluation function $\mathrm{m}_{\mathrm{t}}$ is set to be the sum of trace confidence coefficient. The algorithm formula is derived as follows [6]:

$$
m_{\mathrm{t}}=m_{\mathrm{t}-1}+\Delta m_{\mathrm{t}}
$$

The $d$ represents the distance between the predicted value of the target trajectory and the measured value. $P_{d}$ represents the target detection rate. $\beta_{f}$ represents the density of false alarm space. $M$ represents the dimension of measurement space. Pf represents false alarm rate. $P_{f}=\beta_{f}-V_{k}$. $V_{k}$ represents the volume of the interconnection. Then, the traditional confidence calculation formula is added to the target gray and speed change information as the constraint condition. $\Delta \mathrm{v}_{\mathrm{t}}$ and $\Delta \mathrm{v}_{\mathrm{m}}$ represent the velocity change rate of the trajectory measurement point at t-1 and the velocity change rate of the suspected point at $t$. The $g_{t}$ and $g_{m}$ represent the gray value of the target point at $t-1$ and the gray value of the suspected point at $t$. 


$$
\Delta m_{\mathrm{t}}=\left\{\begin{array}{l}
\frac{\mathrm{c}_{1}}{c_{1}+\left|\Delta v_{t}-\Delta v_{s}\right|} \frac{c_{2}}{c_{2}+\left|g_{t}-g_{s}\right|} \ln \left[\frac{p_{d} e^{\left(-d^{2} / 2\right)}}{\beta_{f}(2 \pi)^{M / 2} \sqrt{|S|}}\right], \text { The trajectory has a measure point update. } \\
\ln \left[\frac{1-p_{d}}{1-p_{f}}\right], \text { No update } \\
m_{1}=\ln \left[\frac{1-\beta_{f}}{\beta_{f}}\right]
\end{array}\right.
$$

According to (10), measured point and target velocity change rate difference, gray value difference, and distance $d$ between the measurement point and the target prediction value, these factors affect the track confidence. The smaller these three values, the greater the confidence coefficient increment.

\section{THE SIMULATION RESULT}

The experiment used the IRST system to acquire multiple frames of continuous infrared images. The image contains multiple interfering motion targets and noise points. Finally, we choose 25 frames of continuous images as sample images, in which all the trajectories changed significantly after the 16th frame. Therefore, this paper mainly introduces the experimental results and results of different algorithms in 16 frames to 25 frames.

Calculation steps and results:

Step1: The MHT method is used to determine the non-zero pixel points in the current frame, and the gray value is compared with the high and low thresholds. The pixel points with gray value higher than the high threshold pixel is added to the tree structure and the root node is created, and the result is marked as table 1 . The pixel points of the gray value between the high and low threshold are added to the tree structure and the root node is created. The result is marked as 0 , and the next frame of image data is waited for further judgment. Discard the pixel gray value less than the low threshold.

Table 1. Examination result

The number of pixels below the low threshold

\begin{tabular}{|c|c|c|c|c|c|c|c|c|c|c|}
\hline No.Pic & 16 th & 17 th & 18 th & 19th & 20th & 21th & 22th & 23th & 24th & 25 th \\
\hline $\begin{array}{c}\text { Number } \\
\text { of pixels }\end{array}$ & 32 & 37 & 56 & 54 & 33 & 19 & 15 & 2 & 6 & 0 \\
\hline
\end{tabular}

The number of possible target pixels

\begin{tabular}{|c|c|c|c|c|c|c|c|c|c|c|}
\hline No.Pic & 16 th & 17 th & 18 th & 19th & 20th & 21th & 22th & 23th & 24th & 25th \\
\hline $\begin{array}{c}\text { Number } \\
\text { of pixels }\end{array}$ & 50 & 56 & 67 & 59 & 57 & 70 & 50 & 41 & 43 & 43 \\
\hline
\end{tabular}

Step2: According to the suspected target pixel obtained in step 1, the system automatically performs connected component division and generates a suspected trajectory. Calculated by the MHT method, 169 suspected trajectories are obtained.

Step3: Using the traditional trace confidence coefficient calculation method, each trace confidence coefficient is calculated and the calculation result is shown in Figure.3(b). Using the adaptive target optimal track extraction method based on target feature constraint optimization, the result is shown in Figure.3(c). 


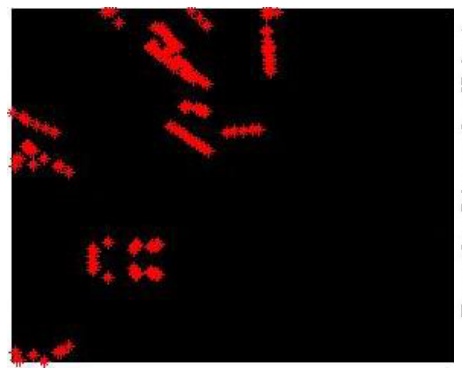

(a)

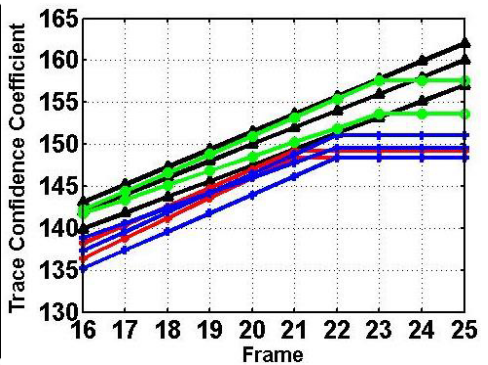

(b)

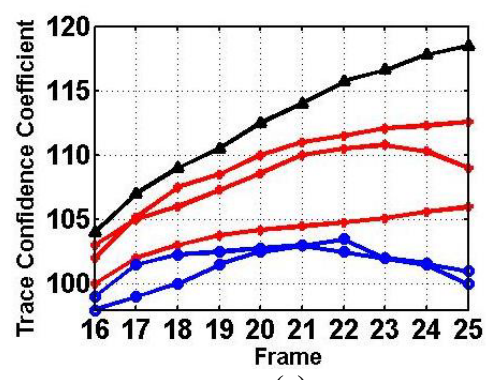

(c)

Figure 3. The result of trace confidence coefficient obtained by two methods

The trajectory obtained using the MHT is shown in Figure. 3(a). Figure. 3(b) is the result of using the traditional trace confidence coefficient calculation method. The difference between true and false trace confidence coefficient is very small. It is difficult to determine the authenticity of the trace. Subsequent data is needed to provide more information to distinguish the true track. According to Figure.3(b), even if the interference target track is associated with the false alarm, its trace confidence coefficient has not changed significantly. According to Figure.3(c), gray value and movement speed of real target are different of other interference target, leading to the obvious change of trace confidence coefficient in continuous frames.

\section{THE CONCLUSION}

This paper introduces the target feature information into the MHT, and proposes the adaptive target optimal track extraction method based on target feature constraint optimization, which improves trace confidence coefficient calculation method and the trajectory starting condition. The simulation results show that the improved method can effectively distinguish the real target trajectory and the interference trajectory in real time, and realize the automatic tracking of small and dim targets in the spacecraft platform.

\section{ACKNOWLEDGMENTS}

This work is supported by the National Natural Science Foundation of China (61671045).

\section{References}

1. R.Chai, Acta Photonica Sinica. J. 33, 1233-1235(2004)

2. D.B.Reid, IEEE Transactions on Automatic Control. J. 24, 843-854 (1980)

3. Biostein.S, D,Huang.T.S, Conference on Computer Vision \& Pattern Recognition. 690-695(1988)

4. W.Wang, Z.Li , J.Liu, Y.He, Z.Chen, Procedia Engineering. J. 29, 1201-1207(2012)

5. L.Liu, Z.Huang, Infrared Physics \& Technology. J. 62, 59-64(2014)

6. Hongren.Zhou, Zhongliang.Jing, Peide.Wang, Maneuvering Target Tracking. (1991) 